\title{
Threshold and magnitude exponent function correlations for the human tongue
}

\author{
LINDA PETROSINO \\ University of North Carolina, Chapel Hill, North Carolina
}

and

\author{
DANIEL HARRIS and DONALD FUCCI \\ Ohio University, Athens, Ohio
}

\begin{abstract}
This study was designed to investigate possible relationships between threshold and suprathreshold lingual tactile processes, and to determine how accurately mean suprathreshold scaling values represent the behavior of individual subjects. Lingual vibrotactile threshold and suprathreshold values were obtained from 26 normal young adults. Results indicated that there was no statistically significant correlation between the threshold and suprathreshold data. It was also determined that mean sensation magnitude values may not accurately represent the behavior of individual subjects.
\end{abstract}

Many early investigations using vibrotactile stimulation to study oral sensory processes focused primarily on measures of lingual vibrotactile thresholds in normal and speech-disordered subjects (Arnst \& Fucci, 1975; Fucci, 1972; Fucci, Blackmon, McCaffrey, \& Lindsey, 1973; Kelly, 1977; Lonegan, 1974; Telage, Fucci, \& Arnst, 1972). The results of these studies suggested that subjects with defective articulation have poorer oral sensory abilities than normal-speaking subjects, and that oral sensory function may be a factor in controlling speech production.

More recently, a series of experiments was initiated to investigate subject behavior in suprathreshold magnitude scaling of vibrotactile stimuli presented to the lingual dorsal surface (Fucci, Harris, \& Petrosino, 1983; Fucci \& Petrosino, 1983a, 1983b, 1983c; Fucci, Petrosino, \& Harris, 1983). These experiments were undertaken to study sensory processes that may operate in the scaling of tactile stimuli presented at controlled levels above threshold. The results of the suprathreshold studies indicated that normal-speaking young adult subjects produced highly stable magnitude scales that appeared to be generated by an internal, absolute scaling mechanism (Fucci et al., 1983). It was also observed that men and women produced similar magnitude scales (Fucci \& Petrosino, 1983c), that increasing age was associated with shallower magnitude function slopes (Fucci \& Petrosino, 1983b), and that stutterers produced steeper magnitude function slopes than did nonstutterers (Fucci, Petrosino, Gorman, \& Harris, 1985).

When viewed collectively, the findings of both the threshold and suprathreshold studies suggest that vibrotactile stimulation is a useful method for investigating the

Please address correspondence to Donald Fucci, School of Hearing and Speech Sciences, Ohio University, Athens, $\mathrm{OH} 45701$. processes involved in obtaining oral tactile thresholds and oral tactile suprathreshold magnitude functions. The purpose of the present study was to investigate possible relationships between threshold and suprathreshold lingual vibrotactile responses and to further determine how accurately mean suprathreshold magnitude exponents represent the behavior of individual subjects.

\section{METHOD}

\section{Subjects}

Twenty-six subjects, 8 men and 18 women, aged 18-28 years (mean $=20.4$ years), were selected randomly from an introductory course in speech and hearing sciences at Ohio University. All subjects had normal speech and hearing and reported no known sensory and/or motor impairments. None of the subjects were experienced in vibrotactile testing.

\section{Apparatus}

The vibrotactile instrumentation consisted of a stimulus unit and a measurement unit. The stimulus unit was composed of a sine-wave generator, an experimenter-controlled $2-\mathrm{dB}$ step variable attenuator, a subjectcontrolled attenuator, two universal timers, an audio amplifier, a power amplifier, a preamplifier, and an electromagnetic minivibrator with a probe-contactor extension. The pulsed vibratory signal generated had a $50 \%$ duty cycle (on $500 \mathrm{msec}$ and off $500 \mathrm{msec}$ ) with a rise-and-decay time of $100 \mathrm{msec}$. The signal frequency was set at $250 \mathrm{~Hz}$. The measurement unit consisted of an accelerometer, a cathode follower, a microphone amplifier, and a voltmeter. A narrow-band noise generator was used to present auditory masking at $70 \mathrm{~dB}$ HTL to the subjects to prevent them from hearing the vibratory stimulus. A detailed description of the instrumentation can be found in an article by Fucci, Petrosino, Wallace, and Small (1982).

\section{Procedure}

All 26 subjects participated in two test sessions with an interval of at least 1 week between sessions. During the first session, each subject was seated in an adjustable chair and positioned so that the anterior dorsum of his or her tongue was in contact with the lower side of a rigidly mounted plastic disk. A hole in the center of the disk provided access 
to the midline of the anterior lingual dorsum for the contactor extension of the vibrator. The contactor had a surface area of $.128 \mathrm{~cm}^{2}$, and there was a 1-mm gap between the contactor and the plastic disk. Each subject's vibrotactile threshold was determined by the ascending method of limits. The accepted threshold was the mean of three successive readings within a 5-mV range. The psychophysical method of magnitude estimation was used to obtain a subjective magnitude function for the lingual dorsum of each subject (Stevens, 1955). The subjects were required to assign numbers to a randomly presented series of nine stimulus intensities ranging from 2 to $40 \mathrm{~dB}$ SL. To minimize possible biases in the results, no reference standard was provided during the task (Hellman \& Zwislocki, 1963). Each subject was instructed to assign a number to each level such that his or her impression of how large the number was matched his or her impression of how intense the vibration was. Whole numbers, decimals, and fractions were designated as permissible selections (Zwislocki \& Goodman, 1980). The geometric means of two runs of the nine stimulus intensities were taken as the magnitude estimation numbers.

During the second experimental session the subjects were positioned as in the first experimental session, and thresholds of sensitivity were established in the same manner as in the first session. The psychophysical method of magnitude production was used to obtain a subjective magnitude function for the lingual dorsum of each subject (Stevens, 1958). The subjects were instructed to use an attenuator $(120-\mathrm{dB}$ variable potentiometer) with a smooth unmarked knob to adjust the amplitude of the stimulus they were feeling on their tongue to "match the magnitude of the number" presented by the experimenter. The experimenter was in control of a master attenuator that could be used to vary the amplitude of the stimulus being delivered to the subject's attenuator. The numbers randomly presented to the subjects were the magnitude estimation values derived from the geometric means of the numbers provided by the subjects during the first experimental session $(0.2,0.5,1.0,2.0$, $3.0,7.0,8.0,10.0)$. For each subject, the geometric means of the millivolt readings for two runs of the magnitude estimation numbers were converted to decibels (SL) and accepted as the magnitude production values.

\section{RESULTS AND DISCUSSION}

The mean thresholds obtained in the magnitude estimation and magnitude production procedures were 2.53 and $2.16 \mathrm{~dB}$, respectively. The mean magnitude exponents obtained for the magnitude estimation and magnitude production procedures were 0.29 and 0.79 , respectively. The threshold-exponent correlations obtained for the magnitude estimation and magnitude production procedures were -0.29 and 0.19 , respectively. The geometric means of the magnitude estimation and magnitude production values for each subject were also calculated in order to counterbalance the data as recommended by Hellman and Zwislocki (1963) and Stevens and Greenbaum (1966). The mean threshold obtained through the magnitude balance procedure was $2.29 \mathrm{~dB}$. The mean magnitude exponent obtained for the magnitude balance procedure was 0.50 . The threshold-exponent correlation obtained for the magnitude balance procedure was -0.06 .

None of the correlations obtained was significant at the .05 alpha level. This result indicates that there was no direct relationship between lingual vibrotactile thresholds and suprathreshold magnitude exponents for the subjects employed in this study. The interpretation of this result is problematic in view of the fact that little similar research is available for comparison. Stevens (1959) and Verrillo and Chamberlain (1972) investigated the relationship be- tween vibrotactile thresholds and magnitude scales at test sites other than the lingual dorsal surface. Stevens (1959) tested the fingertip and the forearm. Verrillo and Chamberlain (1972) tested the fingertip, the thenar eminence of the hand, and the forearm. The results of these studies indicated that sensation magnitude scales appeared to be related to test-site thresholds, with steeper slopes being associated with higher thresholds. Stevens (1959) suggested that this apparent relationship may result from a situation analogous to loudness recruitment in hearing. Vibrotactile test sites with less neural density, just as ears with less functional neural elements would experience a "faster" growth in sensation magnitude once threshold was reached than would a more densely innervated test site.

A direct comparison of the results of the current study with those of Stevens (1959) and Verrillo and Chamberlain (1972) is precluded by the fact that experimental variables such as stimulus frequency, stimulus range, number of sensation levels, contactor size, subject scaling experience, and test sites in those studies were different from those in the present investigation. Also, the present investigation focused on correlations of individual thresholds and magnitude exponents, whereas the previously mentioned research commonly used pooled data to investigate the relationship between mean thresholds and mean magnitude functions.

Several researchers have noted that, when a group of subjects provides matches for the same set of stimulus values, the individual exponents differ significantly from each other (Mitchell \& Gregson, 1971; Teghtsoonian \& Teghtsoonian, 1971, 1983; Wanschura \& Dawson, 1974). Consequently, the assumption that mean magnitude scaling values are representative of the behavior of individual subjects is open to question (Teghtsoonian \& Teghtsoonian, 1983). A distributional analysis was performed on the data from the current study using the SAS (Statistical Analysis System) software package (Ray, 1982) loaded into an IBM 370 Virtual Machine computing system. The results of the analysis are summarized in Table 1 . The coefficients of skewness and kurtosis in Table 1 show that the magnitude exponents were not normally distributed for any of the three experimental methods. The distribution of exponents for magnitude estimation was much flatter than a gaussian distribution, and the distributions for magnitude production and magnitude balance were positively skewed with a steep peak below the mean.

Table 1

Summary of Coefficients of Skewness and Kurtosis From a Distributional Analysis of the Three Psychophysical Methods

\begin{tabular}{ccc}
\hline & \multicolumn{2}{c}{ Coefficients* } \\
\cline { 2 - 3 } Method & Skewness & Kurtosis \\
\hline Magnitude Estimation & 0.14 & -1.22 \\
Magnitude Production & 1.11 & 1.77 \\
Magnitude Balance & 0.91 & 0.28 \\
\hline
\end{tabular}

$* 0=$ Coefficient for Gaussian distribution. 
Histograms of the data for each of the experimental methods provided visual confirmation of the distributions described by the coefficients of skewness and kurtosis. The distributional analysis also showed that the exponents for all three methods were less than 2.6 standard deviations from their respective means, and that none of the sensation magnitude exponents were acting as statistical outliers that could facilitate spurious correlations between exponents and thresholds. The results of the distributional analysis appear to agree with the data of those previously mentioned researchers who concluded that mean sensation magnitude scaling values may not accurately represent the behavior of individual subjects.

\section{REFERENCES}

ARNST, D., \& FUCCI, D. (1975). Vibrotactile sensitivity of the tongue in hearing impaired subjects. Journal of Auditory Research, 15, 115-118.

FuccI, D. (1972). Oral vibrotactile perception: An evaluation of normal and defective speakers. Journal of Speech and Hearing Research, 15, 179-184.

FucCI, D., Blackmon, R., MCCAFFrey, F., \& LindSEY, S. (1973). Oral vibrotactile thresholds of an individual with oral facial anomalies accompanied by severe articulation defects. Ohio Journal of Speech and Hearing, 9, 27-29.

Fucci, D., Harris, D., \& Petrosino, L. (1983). Stability of magnitude production scales for lingual vibrotactile sensation. Perceptual and Motor Skills, 57, 499-504.

FuCCI, D., \& PETrosino, L. (1983a). Lingual vibrotactile sensation magnitudes: Comparison of suprathreshold responses for the tongue and hand. Journal of the Acoustical Society of America, 74, 351-353.

FucCI, D., \& Petrosino, L. (1983b). Lingual vibrotactile sensation magnitudes: Comparison of suprathreshold responses for three different age ranges. Perceptual and Motor Skills, 57, 31-38.

Fucci, D., \& Petrosino, L. (1983c). Lingual vibrotactile sensation magnitudes: Comparison of suprathreshold responses in men and women. Perception \& Psychophysics, 33, 93-95.

Fucci, D., Petrosino, L., Gorman, P., \& Harris, D. (1985). Vibrotactile magnitude production scaling: A method for studying sensoryperceptual responses of stutterers and fluent speakers. Journal of Fluence Disorders, 10, 69-75.
Fucci, D., Petrosino, L., \& Harris, D. (1983). Influence of scaling range on vibrotactile function exponents for the tongue and hand. Perceptual and Motor Skills, 57, 642.

Fucci, D., Petrosino, L., Wallace, D., \& Small, L. (1982). Modification of instrumentation for research on lingual vibrotactile sensitivity: Elimination of the tongue clamping procedure. Review of Scientific Instruments, 53, 1294-1296.

Hellman, R. P., \& Zwislocki, J. (1963). Monaural loudness function at $1000 \mathrm{cps}$ and interaural summation. Journal of the Acoustical Society of America, 35, 856-865.

Kelly, D. (1977). Oral vibrotactile sensation: An evaluation of children with normal and defective articulation. Journal of Communication Disorders, 10, 359-368.

LONEGAN, D. (1974). Vibrotactile thresholds and oral stereognosis in children. Perceptual and Motor Skills, 38, 11-14.

Mitchell, J. J., \& Gregson, R. A. M. (1971). Between-subject variation and within-subject consistency of olfactory intensity scaling. Journal of Experimental Psychology, 89, 314-318.

RAY, A. A. (Ed.). (1982). SAS user's guide: Basics. Cary, NC: SAS Institute, Inc.

STEVENS, S. S. (1955). The measurement of loudness. Journal of the Acoustical Society of America, 27, 815-820.

Stevens, S. S. (1958). Problems and methods of psychophysics. Psychological Bulletin, 54, 177-196.

STEvens, S. S. (1959). Tactile vibration: Dynamics of sensory intensity. Journal of Experimental Psychology, 57, 210-218.

Stevens, S. S., \& Greenbaum, H. B. (1966). Regression effect in psychophysical judgment. Perception \& Psychophysics, 1, 439-446.

Teghtsoonian, M., \& Teghtsoonian, R. (1971). How repeatable are Stevens's power law exponents for individual subjects? Perception \& Psychophysics, 10, 147-149.

Teghtsoonian, M., \& Teghtsoonian, R. (1983). Consistency of individual exponents in cross-modal matching. Perception \& Psychophysics, 33, 203-214.

TelaGe, K., FuCCI, D., \& ARNST, D. (1972). Normative study of oral vibrotactile sensitivity. Perceptual \& Motor Skills, 35, 671-676.

Verrillo, R. T., \& Chamberlain, S. C. (1972). The effect of neural density and contactor surround on vibrotactile sensation magnitude. Perception \& Psychophysics, 11, 117-120.

Wanschura, R. G., \& Dawson, W. E. (1974). Regression effect and individual power functions over sessions. Journal of Experimental Psychology, 102, 806-812.

ZWISLOCKI, J., \& GoodMAN, D. (1980). Absolute scaling of sensory magnitudes: A validation. Perception \& Psychophysics, 28, 28-38.

Manuscript received for publication November 11, 1984.) 